\title{
Cluster Analysis of Data Points using Partitioning and Probabilistic Model-based Algorithms
}

\author{
Ajiboye Adeleke R. \\ Faculty of Computer Systems \\ \& Software Engineering \\ Universiti Malaysia Pahang
}

\author{
Isah-Kebbe Hauwau \\ Faculty of Computer Systems \\ \& Software Engineering \\ Universiti Malaysia Pahang
}

\author{
Oladele Tinuke O. \\ Dept. of Computer Science \\ University of llorin \\ Ilorin, Nigeria.
}

\begin{abstract}
Exploring the dataset features through the application of clustering algorithms is a viable means by which the conceptual description of such data can be revealed for better understanding, grouping and decision making. Some clustering algorithms, especially those that are partitionedbased, clusters any data presented to them even if similar features do not present. This study explores the performance accuracies of partitioning-based algorithms and probabilistic model-based algorithm. Experiments were conducted using kmeans, k-medoids and EM-algorithm. The study implements each algorithm using RapidMiner Software and the results generated was validated for correctness in accordance to the concept of external criteria method. The clusters formed revealed the capability and drawbacks of each algorithm on the data points.
\end{abstract}

\section{General Terms}

Algorithm, Clustering, Data mining

\section{Keywords}

Clustering, Algorithm, K-means, EM-clustering, K-medoids

\section{INTRODUCTION}

Clustering is an important exploratory technique commonly used in descriptive data mining to unveil some hidden features embedded in the dataset. Clustering is described in [1] as an automated search for group of related observations in a data set. The concept involves the division of data into groups, also known as clusters. Although, it is well established that the similarity of objects is used for clustering, the definitions of similarity and the method employed to obtain similarity are varied [2]. The object of the same cluster shares some features, and from a machine learning perspective, clusters correspond to hidden patterns [3]. The clustering algorithm automatically supplies clusters found in data with a conceptual description and according to [4], a good conceptual description can be used for better understanding and better decision. K-means and k-medoids are the most widely used clustering algorithms for selecting group of objects from data sets [5].

Traditionally, clustering techniques are broadly divided into hierarchical and partitioning [3], the notion used in both techniques to cluster data defers. Clustering may also be density or grid-based, while hierarchical algorithm does its grouping as crystals grows, partitioning algorithms learn clusters directly. The hierarchical clustering is further subdivided into agglomerative and divisive.
Clustering is an unsupervised learning technique and unlike classification and regression, which analyse class-labelled data sets, clustering analyses data objects without consulting class labels [6]. It can therefore be used to generate class labels for a group of data. Many similarities exist between data mining and machine learning, but while machine learning research often focuses on the accuracy of the model, data mining research in addition to accuracy places strong emphasis on the efficiency and scalability of mining methods for large data sets; and ways to handle complex types of data and to explore new, alternative methods [6].

This study focuses on comparing the performance accuracies of partitioning-based clustering algorithms and probabilistic model-based clustering of the dataset being explored. The paper is organized as follows: In the next section, some related works reported in the literature on the implementation of these partitioning algorithms and probabilistic model-based clustering is discussed. In section 3 , the basic concepts of each algorithm is briefly discussed; while the processes involved in experimenting with the data is reported in section 4 . The experimental results is represented and discussed in section 5 and the study is concluded in section 6 .

\section{RELATED WORK}

A partitioning-based algorithm such as k-means has been widely reported in the literature for the clustering of data. Specifically, the algorithm is well known for clustering of data such as images [7], video object segmentation [8], document clustering [9] etc. However, one of the drawbacks of the algorithm is its challenges of grouping categorical variables; k-means can only cluster numeric values. The algorithm is sensitive to outliers because such objects are far away from the majority of the data, and thus, when assigned to a cluster, they can dramatically distort the mean value of the cluster [6]. To overcome this challenge, algorithm to address the shortcomings is proposed in [8]. Although, several existing algorithms can handle both numeric and categorical data, Huang[8], opined that most of them are not efficient when dealing with large datasets. In a study proposed in [10], $\mathrm{k}$-means is used to generate class labels; the algorithm was combined with linear discriminant analysis approach to adaptively select the most discriminating subspace.

Study in [11] proposed k-medoids to identify sets of similar rules in order to better understand the pattern of the data. The algorithm is widely proposed in several other studies $[12],[5],[13]$ and[11]. The algorithm was modified in [5] to get a faster clustering and to overcome some of its limitations 
such as the problem of finding natural clusters, the dependency of output on the order of input data, etc. Due to too much time consumed while k-medoids is dealing with very large dataset, the algorithm was scaled in [13] to enhance its performance.

In order to improve the quality of solutions and speed, EM algorithm was enhanced in a study proposed in [14] to cluster large data sets having high dimension. The algorithm is also proposed in [15] for clustering of spatial data. In segmentation of images proposed in [16], the EM algorithm estimates the parameters of the model which provide segmentation of the image into regions, the resulting output produces a description of each region's colour and texture characteristics.

\section{PARTITIONING AND PROBABILISTIC MODEL-BASED ALGORITHMS}

Descriptive algorithms describe features in the dataset based on certain notions. Typical algorithms in this category include: partitioning-based, density-based, grid-based, hierarchical etc. Generally, partitioning-based algorithm finds the partitions or the resulting clusters that minimize either intra-cluster distances or inter-cluster distances. Given a set of $n$ objects, a partitioning method constructs $k$ partitions of the data, where each partition represents a cluster and $k \leq n$; and most partitioning methods are distance-based [6].

\section{1 k-Means}

Suppose a data set, D, contains $n$ objects in Euclidean space, partitioning methods distribute the objects in $\mathrm{D}$ into $k$ clusters, $\mathrm{C}_{1} \ldots, \mathrm{C}_{\mathrm{k}}, \mathrm{C}_{\mathrm{i}} \subset \mathrm{D}$ and $\mathrm{C}_{\mathrm{i}} \cap \mathrm{C}_{\mathrm{j}}=\phi$ for $(1 \leq \mathrm{i}, \mathrm{j} \leq \mathrm{k})$. Kmeans method can only be applied when the mean of a set of objects is defined [6]. A centroid-based partitioning technique uses the centroid of a cluster, $\mathrm{C}_{\mathrm{i}}$ to represent that cluster. The centroid can be defined in various ways, such as by the mean or method of the objects assigned to the cluster. The difference between an object $p \in C i$ and $\mathrm{c}_{\mathrm{i}}$, the representative of the cluster, is measured by $\operatorname{dist}\left(p, \mathrm{c}_{\mathrm{i}}\right)$, where $\operatorname{dist}(\mathrm{x}, \mathrm{y})$ is the Euclidean distance between two points $\mathrm{x}$ and $\mathrm{y}$. The sum of squared error that measures the quality of cluster between all objects in $\mathrm{C}_{\mathrm{i}}$ and centroid $\mathrm{c}_{\mathrm{i}}$, can be defined as:

$\mathrm{E}=\sum_{i=1}^{k} \sum_{p \in C_{i}}^{n} \operatorname{dist}\left(p, c_{i}\right)^{2}$

where $\mathrm{E}$ is the sum of squared error for all objects in the data set; $p$ is the point in space representing a given object; and $\mathrm{c}_{\mathrm{i}}$ is the centroid of cluster $C_{i}$. The time complexity of the $k$ means algorithm is $\mathrm{O}(\mathrm{nkt})$, where $\mathrm{n}$ is the total number of objects, $\mathrm{k}$ is the number of clusters, and $\mathrm{t}$ is the number of iterations [6].

\subsection{EM-algorithm}

In many applications, probabilistic model-based clustering has been shown to be effective, this learning algorithm starts with an initial set of parameters and iterates until the clustering cannot be improved. Generally, the EM- algorithm may not converge to the optimal solution [6]. However, many heuristics have been explored to this situation, most especially by running EM process multiple times using different random initial values. The EM algorithm has two steps: an expectation step and a maximization step; the initial expectation step guesses what the parameters are using pseudo-random numbers. In the maximization step, the mean and variance is used to re-estimate the parameters continually until they converge to a local maximum [17]. Expectation step assigns objects according to the parameters of probabilistic clusters, while maximization step finds the new clustering or expected likelihood in probabilistic model-based clustering [6].

\section{3 k-Medoids}

This algorithm is an extension of k-means paradigm; it clusters categorical data as it uses a simple matching dissimilarity measure for categorical objects [18]. Instead of taking the mean value of the objects in a cluster as a reference point, the actual objects can be picked to represent the clusters, using one representative object per cluster. Each remaining object is assigned to the cluster of which the representative object is the most similar. The partitioning method is then performed based on the principle of minimizing the sum of dissimilarities between each object $p$ and its corresponding representative object. This is the basis for the k-medoids method, which groups $n$ objects into $k$ clusters by minimizing the absolute error criterion[6], which can be defined as:

$$
\mathrm{E}=\sum_{i=1}^{k} \sum_{p \in c_{i}} \operatorname{dist}\left(p, o_{i}\right)
$$

where $E$ is the sum of the absolute error for all objects $\boldsymbol{p}$ in the dataset, and $\mathrm{o}_{\mathrm{i}}$ is the representative object of $\mathrm{c}_{\mathrm{i}}$.

\section{EXPERIMENTATIONS}

This study experiments on excerpts of the dataset retrieved from an open repository of the World Bank [21]. The data reflect the cross-country information for Sector Investment and Capital in the year 2000. Experiment in the proposed study is carried out in the RapidMiner Software environment to describe the features in the data that form the basis for the grouping. The data has three attributes: GDP per capital, urban population and surface area. The target grouping is Income (low, medium, high).

The three partition algorithms used in this study are represented in Table 1 . While k-means measures the Euclidean distance of the data points, k-medoids measures the mixed Euclidean distance of the data points in order to handle string values and with EM-algorithm, data points are

randomly assigned to the parameter $\theta$. In order to compare the cluster results to the label (income group) in the original data, the dataset was clustered into three. Table 1 shows the configuration of the parameters in each algorithm. 


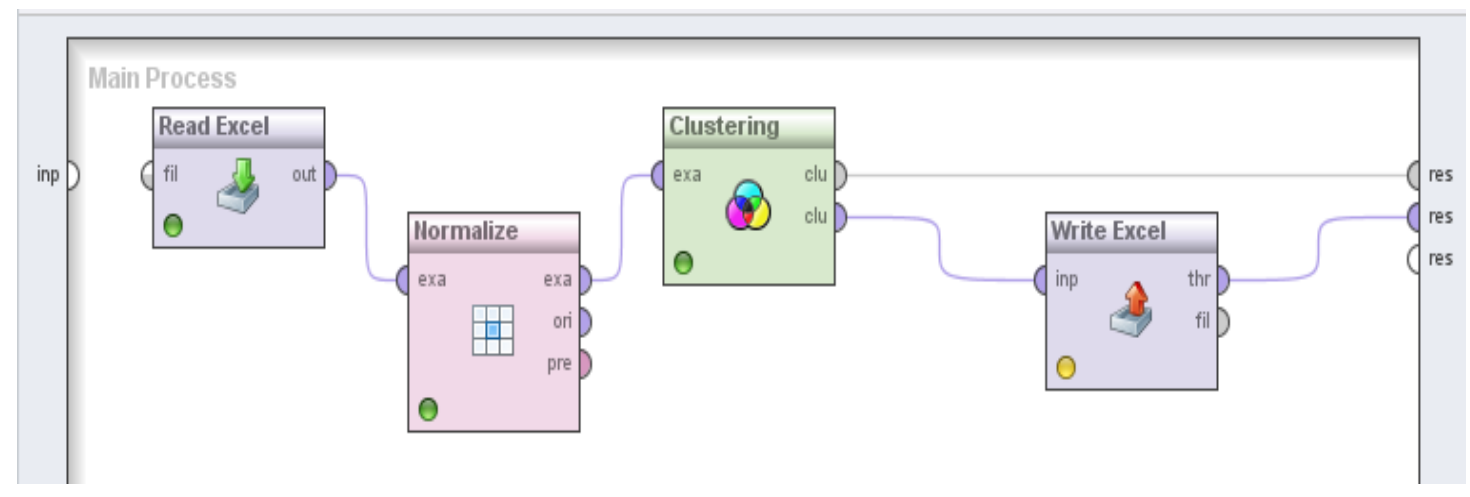

Fig. 1 Experimental setup

Table 1: Parameter settings for the algorithms

\begin{tabular}{|c|c|c|c|c|c|}
\hline Algorithms & $\begin{array}{l}\text { Number of } \\
\text { clusters }\end{array}$ & Max. runs & $\begin{array}{l}\text { Dataset } \\
\text { Normalization }\end{array}$ & $\begin{array}{l}\text { Numerical } \\
\text { measure }\end{array}$ & $\begin{array}{l}\text { Max } \\
\text { optimization }\end{array}$ \\
\hline K-means & 3 & 10 & z-transform & $\begin{array}{c}\text { Euclidean } \\
\text { distance }\end{array}$ & 100 \\
\hline EM-Clustering & 3 & 10 & z-transform & $\begin{array}{c}\text { Assign values } \\
\text { randomly }\end{array}$ & 100 \\
\hline K-medoids & 3 & 10 & z-transform & $\begin{array}{c}\text { Mixed } \\
\text { Euclidean }\end{array}$ & 100 \\
\hline
\end{tabular}

The dataset is normalized in order to express the attributes in smaller units and according to [6], normalized data give attribute greater effect. Normalized and standardized involves transforming the data to fall within small or common range such as $[-1,1]$ or $[0.0,1.0]$. Z-score normalization is a data standardization method that normalized attribute values based on the mean and standard deviation of the attributes values. $A$ value, $X_{i}$ of $A$ is normalized to $\mathrm{X}_{\mathrm{i}}{ }^{1}$ by computing:

$$
X_{i}^{1}=\frac{X_{i}-\bar{A}}{\delta_{A}}
$$

A variation of $\mathrm{z}$-score normalization replaces the standard deviation in (3) by the mean absolute deviation of A. Thus, $\mathrm{z}$-score normalization using the mean absolute deviation is

$$
X_{i}^{1}=\frac{X_{i}-\bar{A}}{S_{A}}
$$

where $\bar{A}$ and $\delta_{A}$ are the mean and standard deviation, respectively, of attribute $A$.

The mean absolute deviation, $\mathrm{S}_{\mathrm{A}}$, is more robust to outliers than the standard deviation, $\delta_{A}[6]$. 
The clustering of data using k-means, EM-algorithm and K-medoids conforms to figures 1, 2 and 3 respectively:

\section{The k-means algorithm}

1. Place $k$ points into the space $\mathrm{S}$

2. Assign each object to the cluster that has the closest centroid

3. Re-compute the positions of the $k$ centroid

4. Repeat steps 2 and 3 until the centroids remain constant.

\section{The EM clustering algorithm}

1. Initialize $\mathrm{i}$ to 0 and choose $\theta_{\mathrm{i}}$ arbitrarily

2. (E-step): Compute $\mathrm{Q}\left(\theta \mid \theta_{\mathrm{i}}\right)$

3. (M-step): Choose $\theta_{\mathrm{i}}+1$ to maximize $\mathrm{Q}\left(\theta \mid \theta_{\mathrm{i}}\right)$

4. If $\theta_{i} !=\theta_{i}+1$, then set $i$ to $i+1$ and return to Step 2

where $\theta$ is an unknown hidden variable.

\section{The k-medoids algorithm}

1. Select the initial medoids

2. Determine the new medoid of each cluster to update medoids

3. Assign each object to the nearest medoid

4. Compute sum of distance from all objects to their medoids

5. Repeat step 2 until the sum remains constant.

Fig. 2: k-means

Fig. 3: EM clustering

Fig. 4: K-medoids

Input: $\quad \mathrm{k}$ : the number of clusters; $\mathrm{S}$ : a data set containing $n$ objects

Output: A set of $k$ clusters

\section{EXPERIMENTAL RESULTS AND DISCUSSION}

This study experiment with two partitioning-based algorithms and a probabilistic model-based algorithm, the clusters formed are shown in Figures 5, 6, and 7. The algorithms operate under similar parameter settings as represented in Table 1. For the purpose of validation of the formed clusters, the results were exported to excel file as shown in the experimental setup in Figure 1 for further computations. The scatter plots of kmeans and k-medoids looks much alike and comparing their results to the class-label of the original data being analysed, each has $61 \%$ and $62 \%$ accuracy respectively as displayed in Table 2. The EM-algorithm shows a different result entirely and the comparisons show that it is $51.8 \%$ accurate. In general, the three algorithms are very fast, while k-means remain the fastest among them.

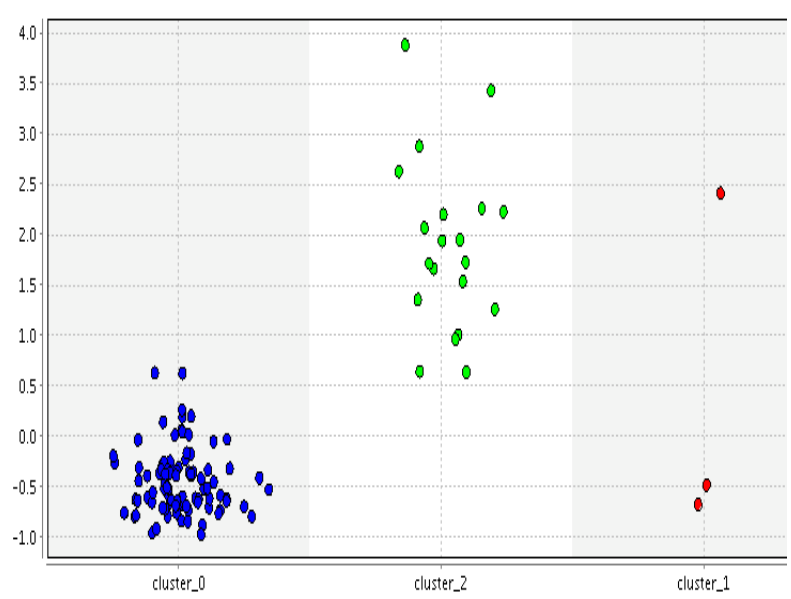

Fig. 5: Scatter plot using k-means algorithm

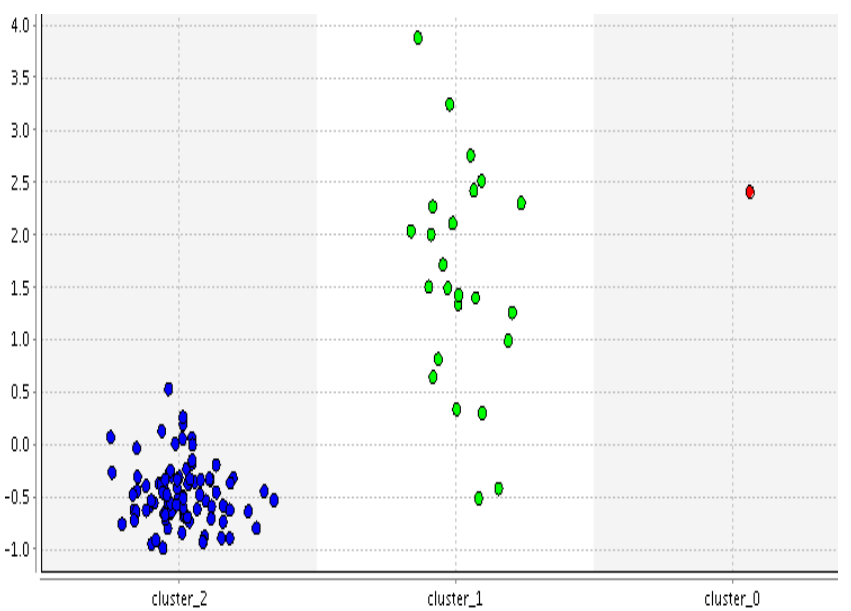

Fig. 6: Scatter plot using k-Medoids algorithm

Table 2: Performance accuracy of each algorithm on the dataset

\begin{tabular}{|l|l|}
\hline Algorithm & Accuracy \\
\hline k-means & $61 \%$ \\
\hline k-medoids & $62 \%$ \\
\hline EM-clustering & $51.8 \%$ \\
\hline
\end{tabular}




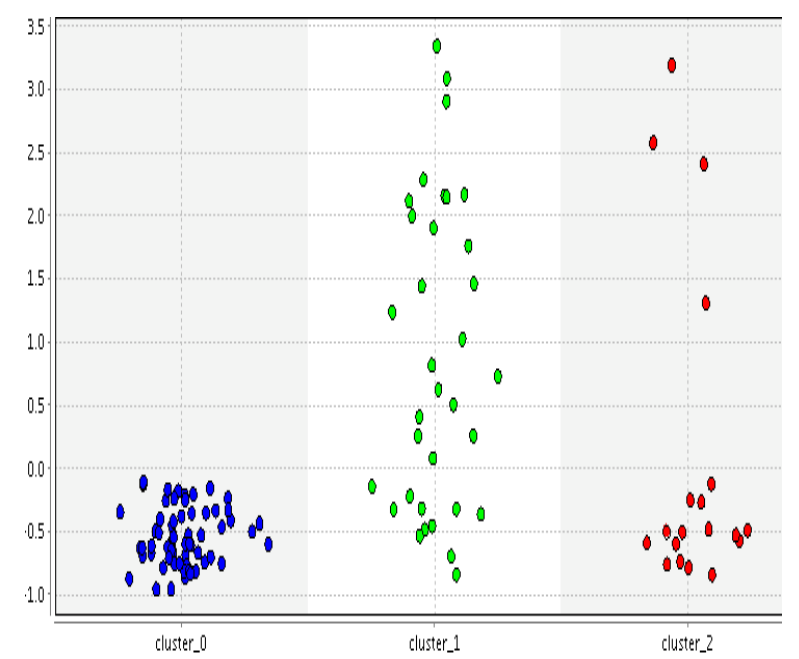

Fig. 7: Scatter plot using EM algorithm

Given a set $X$, the true representative of the set $X[19]$, is a representative set of clustering Centroids $\mathrm{C}_{1}, \ldots, \mathrm{C}_{\mathrm{k}}$ if $|\mathrm{X}|$ $\cap C_{i} \neq \phi$. As reported in [20], the class of an object cannot be predicted by a clustering algorithm, but it may be estimated by examining the clustering result for the class-label data. In order to determine the accuracy of the clusters formed, the class-label of the original data set is mapped to each cluster and the percentage accuracy is determined based on (5).

$\mathrm{S}=$ Sample correctly mapped to the class-label in each cluster

$\mathrm{S}_{\mathrm{T}}=$ Total number of sample data in each cluster

$$
\text { Accuracy }=\sum_{n=1}^{k} \frac{S}{S_{T}} \times 100
$$

where $\mathrm{k}=3$

\section{CONCLUSION}

In this paper, the performance of some partitioning-based clustering algorithms and probabilistic model-based algorithm, namely: k-means, k-medoids and EM-algorithm on structured data are explored with a view to revealing how accurate each algorithm could perform in grouping the dataset. The experimental results are compared to the classlabel of the original data and the performance accuracy of each algorithm displayed in table 2 shows that, k-means and k-medoids are more efficient than EM-algorithm in the clustering of data points. While k-means appear to be the fastest among the three algorithms and is known for its excellent performance on large data, the algorithm however requires that, the value of $k$ be continuously varied to get a cluster of good quality.

\section{REFERENCES}

[1] D. Napoleon and P. G. Lakshmi, "An efficient K-Means clustering algorithm for reducing time complexity using uniform distribution data points," in Trendz in
Information Sciences \& Computing (TISC), 2010, pp. 4245.

[2] S. C. Suh, Practical Applications of Data Mining: Jones \& Barlett Learning, LLC 2012.

[3] P. Berkhin, "A survey of clustering data mining techniques," in Grouping multidimensional data, ed: Springer, 2006, pp. 25-71.

[4] B. Mirkin, Clustering: A Data Recovery Approach: CRC Press, 2012.

[5] G. M. Daiyan, F. Abid, M. Khan, and A. H. Tareq, "An efficient grid algorithm for faster clustering using K medoids approach," in Computer and Information Technology (ICCIT), 2012 15th International Conference on, 2012, pp. 1-3.

[6] J. Han, M. Kamber, and J. Pei, DATA MINING Concepts and Techniques: Morgan Kaufmann, 3rd Edition, 2012.

[7] C.-H. Lin, C.-C. Chen, H.-L. Lee, and J.-R. Liao, "Fast $\mathrm{K}$-means algorithm based on a level histogram for image retrieval," Expert Systems with Applications, vol. 41, pp. 3276-3283, 2014.

[8] Z. Huang, "Extensions to the k-means algorithm for clustering large data sets with categorical values," Data Mining and Knowledge Discovery, vol. 2, pp. 283-304, 1998.

[9] R. Forsati, M. Mahdavi, M. Shamsfard, and M. Reza Meybodi, "Efficient stochastic algorithms for document clustering," Information Sciences, vol. 220, pp. 269-291, 2013.

[10] C. Ding and T. Li, "Adaptive dimension reduction using discriminant analysis and k-means clustering," in Proceedings of the 24th international conference on Machine learning, 2007, pp. 521-528.

[11] A. P. Reynolds, G. Richards, and V. J. Rayward-Smith, "The application of k-medoids and pam to the clustering of rules," in Intelligent Data Engineering and Automated Learning-IDEAL 2004, ed: Springer, 2004, pp. 173-178.

[12] S. M. Razavi Zadegan, M. Mirzaie, and F. Sadoughi, "Ranked $<\mathrm{i}>\mathrm{k}<\mathrm{i}>$-medoids: A fast and accurate rankbased partitioning algorithm for clustering large datasets," Knowledge-Based Systems, vol. 39, pp. 133143, 2013.

[13] R. Joshi, A. Patidar, and S. Mishra, "Scaling k-medoid algorithm for clustering large categorical dataset and its performance analysis," in Electronics Computer Technology (ICECT), 2011 3rd International Conference on, 2011, pp. 117-121.

[14] C. Ordonez and E. Omiecinski, "FREM: fast and robust EM clustering for large data sets," in Proceedings of the eleventh international conference on Information and knowledge management, 2002, pp. 590-599.

[15] C. Ambroise, M. Dang, and G. Govaert, "Clustering of spatial data by the EM algorithm," in geoENV IGeostatistics for environmental applications, ed: Springer, 1997, pp. 493-504.

[16] C. Carson, S. Belongie, H. Greenspan, and J. Malik, "Blobworld: Image segmentation using expectationmaximization and its application to image querying," 
Pattern Analysis and Machine Intelligence, IEEE Transactions on, vol. 24, pp. 1026-1038, 2002.

[17] J. Erman, M. Arlitt, and A. Mahanti, "Traffic classification using clustering algorithms," in Proceedings of the 2006 SIGCOMM workshop on Mining network data, 2006, pp. 281-286.

[18] L. R. Kaufman and P. Rousseeuw, "Finding groups in data: An introduction to cluster analysis," Hoboken NJ John Wiley \& Sons Inc, 1990.

[19] S. Ben-David and M. Ackerman, "Measures of clustering quality: A working set of axioms for clustering," in
Advances in neural information processing systems, 2009, pp. 121-128.

[20] H.-S. Park and C.-H. Jun, "A simple and fast algorithm for K-medoids clustering," Expert Systems with Applications, vol. 36, pp. 3336-3341, 2009.

[21] A Cross-country Database for Sector Investment and Capital - An open repository of the World Bank: http://go.worldbank.org/K955YOONOO (accessed on June $23,2014)$. 